\title{
Equivalence of LP Relaxation and Max-Product for Weighted Matching in General Graphs
}

\author{
Sujay Sanghavi \\ LIDS, MIT \\ sanghavi@mit.edu
}

\begin{abstract}
Max-product belief propagation is a local, iterative algorithm to find the mode/MAP estimate of a probability distribution. While it has been successfully employed in a wide variety of applications, there are relatively few theoretical guarantees of convergence and correctness for general loopy graphs that may have many short cycles. Of these, even fewer provide exact "necessary and sufficient" characterizations.

In this paper we investigate the problem of using max-product to find the maximum weight matching in an arbitrary graph with edge weights. This is done by first constructing a probability distribution whose mode corresponds to the optimal matching, and then running max-product. Weighted matching can also be posed as an integer program, for which there is an LP relaxation. This relaxation is not always tight. In this paper we show that

1) If the $L P$ relaxation is tight, then max-product always converges, and that too to the correct answer.

2) If the $L P$ relaxation is loose, then max-product does not converge.

This provides an exact, data-dependent characterization of maxproduct performance, and a precise connection to LP relaxation, which is a well-studied optimization technique. Also, since LP relaxation is known to be tight for bipartite graphs, our results generalize other recent results on using max-product to find weighted matchings in bipartite graphs.
\end{abstract}

\section{INTRODUCTION}

Message-passing algorithms, like Belief Propagation and its variants and generalizations, have been shown empirically to be very effective in solving many instances of hard/computationally intensive problems in a wide range of fields. These algorithms were originally designed for exact inference (i.e. calculation of marginals/max-marginals) in treestructured probability distributions. Their application to general graphs involves replicating their iterative local update rules on the general graph. In this case however, there are no guarantees of either convergence or correctness in general.

Understanding and characterizing the performance of message-passing algorithms in general graphs remains an active research area. [1,2] show correctness for graphs with at most one cycle. [3,4] show that for gaussian problems the sum-product algorithm finds the correct means upon convergence, but does not always find the correct variances. [5, 6] show asymptotic correctness for random graphs associated with decoding. [7] shows that if max-product converges, then it is optimal in a relatively large "local" neighborhood.

In this paper we consider the problem of using max-product to find the maximum weight matching in an arbitrary graph with arbitrary edge weights. This problem can be formulated as an integer program, which has a natural LP relaxation. In this paper we prove the following

1) If the LP relaxation is tight, then max-product always converges, and that too to the correct answer.

2) If the LP relaxation is loose, then max-product does not converge.

Bayati, Shah and Sharma [8] were the first to investigate max-product for the weighted matching problem. They showed that if the graph is bipartite then max-product always converges to the correct answer. Recently, this result has been extended to $b$-matchings on bipartite graphs [9]. Since the LP relaxation is always tight for bipartite graphs, the first part of our results recover their results and can be viewed as the correct generalization to arbitrary graphs, since in this case the tightness is a function of structure as well as weights.

We would like to point out three features of our work:

1) It provides a necessary and sufficient condition for convergnce of max-product in arbitrary problem instances. There are very few non-trivial classes of problems for which there is such a tight characterization of messagepassing performance.

2) The characterization is data dependent: it is decided based not only on the graph structure but also on the weights of the particular instance.

3) Tightness of LP relaxations is well-studied for broad classes of problems, making this chracterization promising in terms of both understanding and development of new algorithms.

Relations, similarities and comparisons between max-product and linear programming have been used/mentioned by several authors [10-12], and an exact characterization of this relationship in general remains an interesting endeavor. In particular, it would be interesting to investigate the implications of these results as regards elucidating the relationship between iterative decoding of channel codes and LP decoding [13].

\section{Weighted MATCHING AND ITS LP RELAXATION}

A matching in a graph is a set of edges such that no two edges in the set are incident on the same node. Given a graph $G=(V, E)$, with non-negative weights $w_{e}$ on the edges $e \in$ $E$, the weighted matching problem is to find the matching $M^{*}$ whose edges have the highest total weight. In this paper we find it convenient to refer to edges both as $e \in E$ and as $(i, j)$, where $i, j \in V$. 
Weighted matching can be written as the following integer program (IP):

$$
\begin{gathered}
\max \quad \sum w_{e} x_{e} \\
\text { s.t. } \sum_{j \in \mathcal{N}(i)} x_{i j} \leq 1 \quad \text { for all } i \in V \\
x_{e} \in\{0,1\} \quad \text { for all } e \in E
\end{gathered}
$$

The LP relaxation of the above problem is to replace the constraint $x_{e} \in\{0,1\}$ with the constraint $x_{e} \geq 0$. This relaxation is in general not tight, i.e. there might exist noninteger solutions with strictly higher value than any integral solution. It is known however that the LP relaxation is always tight for bipartite graphs: no matter what the edge weights, the bipartite-ness ensures tightness of the LP relaxation. If a graph is not bipartite, the tightness of the LP relaxation will depend on the edge weights: the same graph may have tightness for one set of weights and looseness for another set.

The dual of the above linear program is the vertex cover problem: minimize the total of the weights $z_{i}$ that need to be placed on nodes so as to "cover" the edge weights: (DP)

$$
\begin{gathered}
\min \sum z_{i} \\
\text { s.t. } \quad w_{i j} \leq z_{i}+z_{j} \quad \text { for all }(i, j) \in E \\
z_{i} \geq 0 \quad \text { for all } i
\end{gathered}
$$

Lemma 1 (complimentary slackness): When the LP relaxation is tight, the optimal matching $M^{*}$ and the optimal dual variables $z$ and satisfy the following properties:

1) if $(i, j) \in M^{*}$ then $w_{i j}=z_{i}+z_{j}$

2) if $(i, j) \notin M^{*}$ then $w_{i j} \leq z_{i}+z_{j}$

3) if no edge in $M^{*}$ is incident on node $i$, then $z_{i}=0$

4) $z_{i} \leq \max _{e} w_{e}$ for all $i$

\section{BACKGRound on the MAX-Product Algorithm}

The factor graph [14] of a probability distribution represents the conditional independencies of the distribution. The MaxProduct (MP) algorithm is a simple, local, iterative message passing algorithm that can be used (in an attempt) to find the mode/MAP estimate of a probability distribution. Nodes and factors pass messages to each other, and nodes maintain "beliefs", which represent the max-marginals. When maxproduct is applied to problems involving general "loopy" graphs, one of the following three scenarios may result:

1) The algorithm may not converge.

2) The algorithm may converge, but to an incorrect answer.

3) The algorithm may converge to the correct answer.

As has been mentioned, here has been siginifcant work attempting to understand the properties of MP for loopy graphs. For the results in this paper, we will use the following two insights:

1) At any time, the belief of the max-product algorithm for a given variable corresponds to the belief at the root of the corresponding computation tree distribution [2] associated with that variable at that time. We describe what this computation tree distribution corresponds to for the weighted matching problem in the next section.

2) If max-product does converge, the resulting beliefs are optimal in a large "local" neighborhood [7]: let $\widehat{x}$ be the assignment as given by the converged max-product and $\widetilde{x}$ be any other assignment. If the variables assigned different values in $\widehat{x}$ and $\widetilde{x}$ form an induced graph containing at most one cycle in each component, then $p(\widehat{x}) \geq p(\widetilde{x})$.

\section{MaX-Product For Weighted Matching}

The problem of finding $M^{*}$ can be formulated as the problem of finding the mode of a suitably (artifically) constructed probability distribution $p$. In fact, there are in general several ways to construct this distribution for the same instance of a graph $G$. We now present one construction! 1 .

Associate a binary variable $x_{e} \in\{0,1\}$ with each edge $e \in E$, and let

$$
p(x)=\frac{1}{Z} \prod_{i \in V} \mathbf{1}_{\left\{\sum_{j \in \mathcal{N}(i)} x_{i j} \leq 1\right\}} \prod_{e \in E} e^{w_{e} x_{e}}
$$

Here $\mathcal{N}(i)$ represents the neighborhood of node $i$ in $G$, and $Z$ is a normalizing constant. The variable $x_{e}$ can be interpreted as follows: $x_{e}=1$ indicates that $e \in M^{*}$, while $x_{e}=0$ indicates $e \notin M^{*}$. The term $\mathbf{1}_{\left\{\sum_{j \in \mathcal{N}(i)} x_{i j} \leq 1\right\}}$ enforces the cosntraint that of the edges incident to node $i$, at most one can be assigned the value " 1 ". Thus, it is easy to see that $p(x)>0$ if and only if the edges with $x_{e}=1$ constitute a matching in $G$. Furthermore, the mode of $p$ corresponds to the max-weight matching $M^{*}$.

The factor graph max-product involves messages between variables and factors. In our case the variables are the edges $(i, j) \in E$, and the factors are nodes $i \in V$. Thus at any time $t$ there will be messages $m_{i \rightarrow(i, j)}^{t}$ from node (factor) $i$ to edge (variable) $(i, j)$, as well as messages $m_{(i, j) \rightarrow i}^{t}$. Each message will be a length-two vector of real numbers, indexed by 0 and 1 . The message update rules can be simplified to the following:

$$
\begin{aligned}
& m_{(i, j) \rightarrow i}^{t+1}[1]=e^{w_{i j}} m_{j \rightarrow(i, j)}^{t}[1] \\
& m_{(i, j) \rightarrow i}^{t+1}[0]=m_{j \rightarrow(i, j)}^{t}[0] \\
& m_{i \rightarrow(i, j)}^{t+1}[1]=\prod_{k \in \mathcal{N}(i)-j} m_{(k, i) \rightarrow i}^{t}[0] \\
& m_{i \rightarrow(i, j)}^{t+1}[0]=\max \left\{\prod_{k \in \mathcal{N}(i)-j} m_{(k, i) \rightarrow i}^{t}[0]\right. \\
& \left.\max _{k \in \mathcal{N}(i)-j} m_{(k, i) \rightarrow i}^{t}[1]\right\}
\end{aligned}
$$

Also, at every time each edge (variable) maintains a belief vector $b_{(i, j)}^{t}$ as follows:

$$
\begin{aligned}
& b_{(i, j)}^{t}[0]=m_{i \rightarrow(i, j)}^{t}[0] \times m_{j \rightarrow(i, j)}^{t}[0] \\
& b_{(i, j)}^{t}[1]=e^{w_{i j}} m_{i \rightarrow(i, j)}^{t}[1] \times m_{j \rightarrow(i, j)}^{t}[1]
\end{aligned}
$$

\footnotetext{
${ }^{1}$ This construction is different from the one in [8], which had a pairwise model with variables corresponding to nodes in the graph. However, the results of this paper continue to hold when the construciton in [8] is modified to be applicable to general graphs
} 
The $p$ defined above can be used to find $M^{*}$ as follows: first run max-product. At any time $t$ and for each edge $e$ there will be two beliefs $b_{e}^{t}[0]$ and $b_{e}^{t}[1]$. If max-product converges, assign to each variable the value (i.e. " 0 " or " 1 ") that corresponds to the stronger belief. Then, declare the set of all edges set to " 1 " to be the max-product output.

\section{A. The Computation Tree for Weighted Matching}

Our proofs rely on the computation tree interpretation [2, 15] of the Max-product beliefs. We now describe this interpretation when max-product is applied to $p$ as given in (2).

For an edge $e$ let $\bar{T}_{e}(k)$ be the full depth- $k$ computation tree rooted at $e$. This is generated recursively: take $\bar{T}_{e}(k-1)$ and to each leaf $v$ add as children a copy of each of the neighbors of $v$ in $G$, except for the unique neighbor of $v$ which is already present in $\bar{T}_{e}(k-1)$. Also, each new edge has the same weight as its copy in the original $G$. The recursion is started with the single-edge tree $\bar{T}_{e}(1)=e$, both of whose endpoints are leaves. This initial edge is the root of $\bar{T}_{e}$.

Consider now the "full synchronous" max-product, where at each time every message in the network is updated. In this case the computation tree $T_{e}(k)$ for edge $e$ at time $k$ will be $\bar{T}_{e}(k)$. Alternatively, max-product may be executed asynchronously with only a subset of the messages updated in every time slot. In this case $T_{e}(k)$ will be a sub-tree of $\bar{T}_{e}(k)$. In either case, the computation tree interpretation states at time $k$ we have $b_{e}^{k}[1]>b_{e}^{k}[0]$ if and only if the root of $T_{e}(k)$ is a member of a max-weight matching on the tree $T_{e}(k)$.

The figure below shows an example where on the left is $G$ : the four-cycle $a b c d$ and the chord $a c$, with a matching $M=\{(a, b),(c, d)\}$ depicted in bold. On the right is the computation tree $\bar{T}_{(a, b)}(4)$ which is the full tree of depth 4 rooted at edge $(a, b)$. The bold edges depict the projection $M_{T}$ of $M$ onto $\bar{T}_{(a, b)}(4):$ an edge $e$ in the tree is in $M_{T}$ if and only if its copy in $G$ is in $M$.
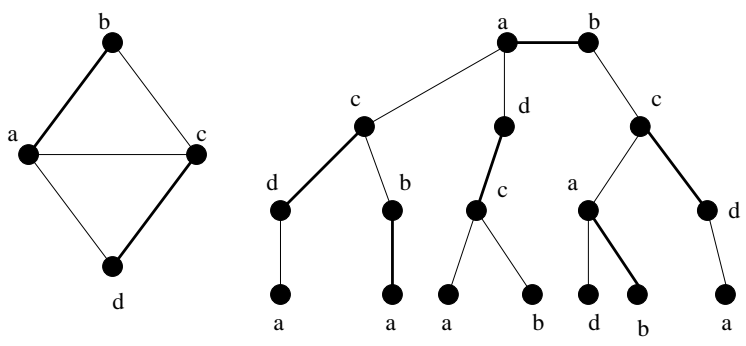

Lemma 2: Let $M$ be a matching in $G$ and $T_{e}(k)$ be a computation tree. Let $M_{T}$ be the set of all copies in $T_{e}(k)$ of all edges in $M$. Then, $M_{T}$ is a matching in $T_{e}(k)$. Also, if $M$ is maximal in $G, M_{T}$ is maximal in $T_{e}$.

Of course $T_{e}$ will also contain other matchings that are not projections of matchings in $G$. Finally, we say that a (possibly not full) tree $T_{e}(k)$ is full upto depth $k_{1}$ if the full tree $\bar{T}_{e}\left(k_{1}\right)$ is contained in $T_{e}(k)$.

\section{Equivalence of MAX-Product ANd LP RELAXATION}

We are now ready to prove the main result of this paper: the equivalence of Max-Product and LP Relaxation. Before we proceed, we define the following terms

1) We say that the LP relaxation is tight if the linear program (LP) obtained by relaxing the integer program (1) has a unique optimal solution at which all values $x_{e}$ are either 0 or 1 .

2) We say that max-product converges by step $k$ if the variable assignments ( 0 or 1$)$ that maximize the beliefs at each node remain constant once the associated computation tree is full up to depth at least $k$. Note that this includes both synchronous and asynchronous message updates. We say that max-product converges if there exists some $k<\infty$ such that max-product converges by step $k$. Finally, we say that max product converges to the correct answer if the beliefs $b_{e}$ at convergence are such that $b_{e}[1]>b_{e}[0]$ if and only if $e \in M^{*}$, and $b_{e}[1]<b_{e}[0]$ if and only if $e \notin M^{*}$

We also need to make some uniqueness assumptions. It is well-recognized that max-product may perform poorly in the presence of multiple optima, and that characterizing performance in this case is hard. For the rest of this paper we will assume the following:

A1 $M^{*}$ is the unique optimal matching.

A2 The linear program always has a unique optimal solution. Note that this can be fractional, but it has to be unique.

\section{A. Max-product is as Powerful as LP Relaxation}

In this section we prove that if the LP relaxation is tight then Max-Product converges to the correct answer. Recall that when the LP is tight, part 2 of Lemma 1 says that if $(i, j) \notin M^{*}$ then $w_{i j} \leq z_{i}+z_{j}$. The uniqueness assumptions A1-2 further imply that the inequality is strict: $w_{i j}<z_{i}+z_{j}$. Another way of saying this is that there exists an $\epsilon>0$ such that

$$
w_{i j} \leq z_{i}+z_{j}-\epsilon \text { for all }(i, j) \notin M^{*}
$$

Theorem 1: Consider a weighted graph $G$ for which the LP relaxation is tight. Then max-product converges to the correct answer by step $\frac{2 w_{\max }}{\epsilon}$, where $w_{\max }=\max _{e} w_{e}$ is the weight of the heaviest edge, and $\epsilon$ satisfies (3).

Proof:

Let $M^{*}$ be the optimal matching on $G$. For max-product to be convergent and correct, we need that $b_{e}^{t}[1]>b_{e}^{t}[0]$ for all $e \in M^{*}$ and $b_{e}^{t}[1]<b_{e}^{t}[0]$ for all $e \notin M^{*}$, and for all $t$ such that $T_{e}(t)$ is full upto depth $\frac{2 w_{\max }}{\epsilon}$.

So suppose that for such a $t$ there exists an $e \notin M^{*}$ such that $b_{e}^{t}[1]>b_{e}^{t}[0]$. Then, there exists a matching $M$ in $T_{e}(t)$ such that (a) the root $e \in M$, and (b) $M$ has the largest weight among matchings on $T_{e}(t)$. Let $M_{T}^{*}$ be the set of all edges in $T_{e}(t)$ that are copies of edges in $M^{*}$. By lemma $2, M_{T}^{*}$ is a maximal matching on $T_{e}(t)$. Also, the root $e \notin M_{T}^{*}$ by assumption. 
The symmetric difference $M_{T}^{*} \triangle M$ consists of disjoint alternating paths in $T_{e}(t)$ : each path will have every alternate edge in $M_{T}^{*}$ and all other edges in $M$. Let $P$ be the path that contains the root $e$. We now show that $w\left(P \cap M_{T}^{*}\right)>$ $w(P \cap M)$.

Recall that the optimal dual solution assigns to each node $i$ in $G$ a "dual value" $z_{i} \geq 0$. Associate now with each node in $T_{e}(t)$ the dual value of its copy in $G$. Then, by Lemma 1 we have that $w_{i j}=z_{i}+z_{j}$ for each $(i, j) \in P \cap M_{T}^{*}$. Suppose now that neither endpoint of $P$ is a leaf of $T_{e}(t)$. In this case, we have

$$
w\left(P \cap M_{T}^{*}\right)=\sum_{i \in P} z_{i}
$$

On the other hand, we know that (3) holds for each edge in $P \cap M$. Adding these up gives

$$
w(P \cap M) \leq \sum_{i \in P} z_{i}-\epsilon|P \cap M|
$$

By assumption, the root $e \in P \cap M$, so $|P \cap M| \geq 1$ and hence $w\left(P \cap M_{T}^{*}\right)>w(P \cap M)$ when no endpoints of $P$ are leaves.

Suppose now that exactly one of the endpoints $v$ of $P$ is a leaf of $T_{e}(t)$. In this case, we have that

$$
w\left(P \cap M_{T}^{*}\right) \geq \sum_{i \in P} z_{i}-z_{v} \geq \sum_{i \in P} z_{i}-w_{\max }
$$

where the last inequality follows from part 4 of Lemma 11 Also, $T_{e}(t)$ is assumed to be full up to depth $k$, so this implies that $|P \cap M| \geq \frac{k}{2}$. This means that

$$
w(P \cap M) \leq \sum_{i \in P} z_{i}-\epsilon \frac{k}{2}
$$

Now, since $k \geq \frac{2 w_{\max }}{\epsilon}$, this implies that $w\left(P \cap M_{T}^{*}\right)>w(P \cap$ $M)$. The final case, where both endpoints of $P$ are leaves, works out in the same way, except that now $|P \cap M| \geq k$ and $w\left(P \cap M_{T}^{*}\right) \geq \sum_{i \in P} z_{i}-2 w_{\max }$.

Thus, in any case, we have that $w\left(P \cap M_{T}^{*}\right)>w(P \cap M)$. Consider now the set of edges $M-(P \cap M)+\left(P \cap M_{T}^{*}\right)$. This set forms a matching on $T_{e}(t)$, and has higher weight than $M$. This contradicts the choice of $M$, and so establishes that $b_{e}^{t}[1]<b_{e}^{t}[0]$ for all $e \notin M^{*}$. A similar contradiction argument can be used to establish that $b_{e}^{t}[1]>b_{e}^{t}[0]$ for all $e \in M^{*}$. This completes the proof.

\section{B. LP Relaxation is as Powerful as Max-product}

In this section we prove that if the LP relaxation is loose then max-product does not converge to the correct answer. Before we do so however, we note that this implies a stronger result: that when LP is loose then in fact max-product does not converge at all.

Lemma 3: Consider the distribution $p(x)$ as given in (2). If Max-Product converges, then its output exactly corresponds to the true optimal matching $M^{*}$.

The proof of this lemma uses the "local optimality" result of Weiss and Freeman [7]. In particular, for $p$ it turns out that local optimality implies global optimality. This means that it is not possible for max-product to converge to an incorrect answer: it will either not converge at all, or converge to $M^{*}$. We do not use this explicitly in the proofs below, but it strengthens the results as mentioned above.

We now proceed with showing that max-product does not converge to the correct $M^{*}$ when LP is loose. As a first step, we need a combinatorial characterization of when the LP relaxation is loose. We now make some definitions. We say that a node $v$ is saturated by a matching $M$ if there exists an edge $e \in M$ that is incident to $v$.

A blossom with respect to a matching $M$ is an odd cycle $C$ with $\frac{|C|-1}{2}$ edges in $M \sqrt{2}$ Note that a blossom has a unique base: a node not saturated by any edge in $C \cap M$. A stemmed blossom $B_{1}$ (w.r.t $M$ ) is a blossom $C$, along with an alternating path (stem) $P$ that starts at the base of $C$, and starts with an edge in $M$. Also, $P$ should be such that the set $M-(P \cap$ $M)+(P-M)$ remains a matching in $G$.

A bad stemmed blossom is one in which the edge weights satisfy

$$
w(C \cap M)+2 w(P \cap M)<w(C-M)+2 w(P-M)
$$

Note that it may well be the case that $|P|=0$, in which case $B_{1}$ is just an odd cycle. The following is an example of a bad stemmed blossom. The bold edges are the ones in $M$, the numbers denote the weights of the corresponding edges, and the last node $i$ has no edge of $M$ incident on it. The blossom $C$ in this case is the cycle $a b c d e$, and node $c$ is its base. The path/stem $P$ is $c f g h i$.

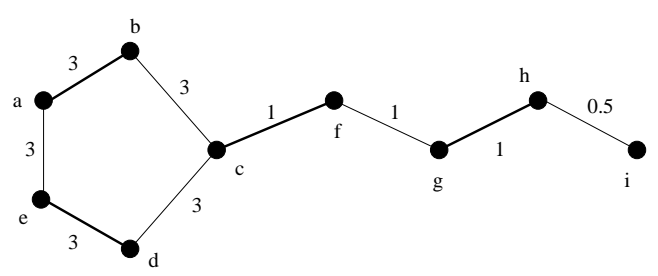

A blossom pair $B_{2}$ is two blossoms $C_{1}$ and $C_{2}$ and an alternating path $P$ between the bases of the two blossoms such that $P$ begins and ends with edges in $M$. A bad blossom pair is one in which the edge weights satisfy

$$
\begin{aligned}
& w\left(C_{1} \cap M\right)+w\left(C_{2} \cap M\right)+2 w(P \cap M) \\
& \quad<w\left(C_{1}-M\right)+w\left(C_{2}-M\right)+2 w(P-M)
\end{aligned}
$$

The following is an example of a bad blossom pair.

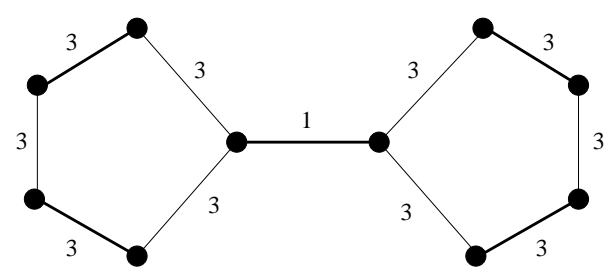

\footnotetext{
${ }^{2}$ Blossoms were first defined in [16], which also provided the first efficient algorithm for weighted matching in arbitrary graphs.
} 
The following proposition provides a combinatorial characterization of when the LP relaxation is loose, and is crucial to the proof of the subsequent theorem.

Proposition 1: If the LP relaxation is loose, then there exists a bad stemmed blossom, or a bad blossom pair, with respect to the optimal matching $M^{*}$.

Proof: In appendix.

We use the presence of these "bad" subgraphs in $G$ to show that max-product does not converge to the correct answer. Before we do so, we need one additional lemma. This states that if max-product converges by step $k$ to some matching $M$ on $G$, then the optimal matching $M_{T}$ on the computation tree looks like $M$ in the neighborhood of the root.

Lemma 4: Suppose max-product converges to a matching $M$ in $G$ by step $k$. Consider any edge $e$, some $m \geq 1$ and a corresponding computation tree $T_{e}$ which is full up to depth $k+m$. Let $M_{T}$ be the max-weight matching on the tree. Then, for any edge $f \in T_{e}$ that is within distance $m$ of the root $e$, $f \in M_{T}$ if and only if its copy $f_{1}$ in $G$ is such that $f_{1} \in M$.

Note that the above lemma also applies to the root $e$ of the tree. We are now ready to state and prove the main result of this section. Recall that the belief $b_{e}$ on an edge at convergence is incorrect if either $e \in M^{*}$ but $b_{e}[0]>b_{e}[1]$, or $e \notin M^{*}$ but $b_{e}[1]>b_{e}[0]$.

Theorem 2: Consider a weighted graph $G$ for which the LP relaxation is loose. Then, the max-product beliefs do not converge to the correct $M^{*}$ : for any given $k$, there exists a $k_{1} \geq k$ and computation trees $T_{e}, e \in E$ such that each $T_{e}$ is full upto depth $k_{1}$, but the beliefs on some of the edges are incorrect. Lemma 3 further implies that in fact in this case max-product does not converge at all.

Proof:

Let $M^{*}$ be the max-weight matching on $G$. Since the LP relaxation is loose, by Prop. 11 there exists either a bad stemmed blossom or a bad blossom pair w.r.t. $M^{*}$. Suppose first that it contains a bad stemmed blossom $B_{1}$, and consider some $e \in C \cap M^{*}$ that is in the "blossom" part of $B_{1}$ (as opposed to the stem) and also in $M^{*}$. From the two nodes of $e$, make maximal alternating paths $P_{1}$ and $P_{2}$ that remain in $B_{1}$ and start out in opposite directions on $C$. For the stemmed blossom example above, if $e$ is the edge $(a, b)$ then the two paths will be bcfghi and aedcfghi.

Let $d_{1}=w\left(P_{1}-M^{*}\right)-w\left(P_{1} \cap M^{*}\right)$, and similarly $d_{2}$ for $P_{2} . d_{1}$ represents the change in the weight of the matching if each edge in $P_{1}$ were "switched", i.e. their membership in the matching was reversed from its original value. It is easy to see that

$$
\begin{aligned}
d_{1}+d_{2}-w(e)=\quad w\left(C-M^{*}\right)+2 w\left(P-M^{*}\right) \\
-w\left(C \cap M^{*}\right)-2 w\left(P \cap M^{*}\right)
\end{aligned}
$$

By assumption $B_{1}$ is a bad blossom and hence we have that $d_{1}+d_{2}-w(e)>0$.

Suppose max-product converges to $M^{*}$ by step $k$. Consider now the computation tree $T_{e}$ which is full upto depth $k+$ $|V|$, where $|V|$ is the number of nodes in $G$. Let $M_{T}$ be the max-weight matching on $T_{e}$. Lemma 4 implies that $M_{T}$ will be a projection of $M^{*}$ in a distance- $|V|$ neighborhood of the root. Also, starting from the root $e$, each of $P_{1}$ and $P_{2}$ will have a unique copy, say $R_{1}$ and $R_{2}$ respectively, in $T_{e}$, with $\left|R_{1}\right|,\left|R_{2}\right|<|V|$. Since $P_{1}$ and $P_{2}$ are alternating w.r.t. $M^{*}$, it follows that $R_{1}$ and $R_{2}$ will be alternating with respect to $M_{T}$. Also, the set $S=R_{1} \cup e \cup R_{2}$ forms an alternating path on $T_{e}$ with respect to $M_{T}$, and this begins and ends in nodes unsaturated by $M_{T}$. Thus, $M_{T}$ can be augmented by this path: the set $M_{T}-\left(S \cap M_{T}\right)+\left(S-M_{T}\right)$ will be a matching on $T_{e}$.

Also, the weight gain from doing this augmentation will be exactly $d_{1}+d_{2}-w(e)$, which we know is strictly positive. Thus, this shows that $M_{T}$ is not the optimal matching on $T_{e}$, which contradicts the choice of $M_{T}$. This means that our assumption about max-product convergence to $M^{*}$ is incorrect.

Thus, we see that if there exists a bad stemmed blossom w.r.t. $M^{*}$ in $G$ then max-product does not converge to $M^{*}$. A similar argument holds for the case of a bad blossom pair $B_{2}$, except that instead of paths $P_{1}$ and $P_{2}$ above we now have to look at alternating walks $W_{1}$ and $W_{2}$ that live in $B_{2}$ and are long enough. These walks can then be mapped to an augmenting path on $T_{e}$ which strictly improves $M_{T}$, leading to a contradiction as was seen in the case of the paths $P_{1}$ and $P_{2}$. This completes the proof.

\section{DISCUSSION}

The results of this paper can be generalized to the case of perfect matchings, $b$-matchings and perfect $b$-matchings in general graphs, where similar results hold. In this paper maxproduct is shown to be as powerful as LP relaxation, but it would be more interesting to outline a direct operational link between max-product and a linear programming algorithm. As an example, [8] shows that for bipartite matching max-product has an operational correspondance with the auction algorithm [17]. Also, the form of the message update equations suggests that it can be implemented via an equivalent message passing update rule between just the nodes of the graph $G$, instead of having messages go from nodes to edges and vice versa.

More generally, it would be interesting to see if the ideas presented in this paper could be used/genealized to show connections between linear programming and belief propagation in other applications.

\section{ACKNOWLEDGEMENTS}

The author would like to acknowledge Dmitry Malioutov, whose experiments suggested a strong link between LP relaxation and max-product performance for non-bipartite graphs. Dmitry is also responsible for pointing the author to the local optimality result [7].

\section{APPENDIX}

\section{Proof of Proposition 1}

We now show that if the LP relaxation is loose then there exists in the graph either a bad stemmed blossom or a bad 
blossom pair, with respect to the optimal matching $M^{*}$. Let $x$ be the optimal (fractional) solution to the LP relaxation.

Let $E^{\prime}$ be the set of all edges $e$ such that either $(a) e \in M^{*}$, or $(b) e \notin M^{*}$ and $x_{e}>0$. Then, $E^{\prime}$ will contain at least one edge $e \notin M^{*}$, because if all $e \notin M^{*}$ had $x_{e}=0$ then the LP would be tight. Let $G^{\prime}=\left(V, E^{\prime}\right)$ be the subgraph of $G$ having only the edges in $E^{\prime}$. An cycle augmentation is any even cycle in which every alternate edge is in $M^{*}$. A path augmentation is any path in which every alternate edge is in $M^{*}$, and which begins and ends in nodes unsaturated by $M^{*}$. For any augmentation $A$, we have that $M^{*}-\left(A \cap M^{*}\right)+(A-$ $\left.M^{*}\right)$ is also a matching in $G^{\prime}$. Thus, if $M^{*}$ is the unique maxweight matching it has to be that $w\left(A \cap M^{*}\right)>w\left(A-M^{*}\right)$.

Lemma 5: $G^{\prime}$ cannot contain any augmentations: cycles or paths.

Proof: Let $A$ be an augmentation in $G^{\prime}$. By assumption, $x_{e}>0$ for all $e \in A-M^{*}$, which implies that $x_{e}<1$ for all $e \in A \cap M^{*}$. Thus, there exists some $\epsilon>0$ such that decreasing each $x_{e}, e \in A-M^{*}$ by $\epsilon$ and increasing each $x_{e}, e \in A \cap M^{*}$ by $\epsilon$ represents a valid new feasible point for the LP. The weight of this new point exceeds the weight of $x$ by $\epsilon\left(w\left(A \cap M^{*}\right)-w\left(A-M^{*}\right)\right)>0$. However this contradicts the optimality of $x$, and thus $G^{\prime}$ cannot contain any augmentation.

Let $S$ be the longest alternating sequence of edges in $G^{\prime}$, and let $v_{1}$ and $v_{2}$ be its endpoints. By the lemma above, both cannot be unsaturated. We say that $v_{1}$ or $v_{2}$ is a saturated leaf if it is saturated by $M^{*}$ and there exist no edges in $G^{\prime}-M^{*}$ incident on it. Also, note that an endpoint is saturated if and only if its corresponding edge in $S$ is also in $M^{*}$.

The fact that $S$ is the longest sequence means that it cannot be extended further beyond $v_{1}$ and $v_{2}$. This implies that one of the following cases must occur:

1) Both $v_{1}$ and $v_{2}$ are both saturated leaves

In this case, the constraints at $v_{1}$ and $v_{2}$ are loose. So, there exists an $\epsilon$ such that if all $x_{e}, e \in S-M^{*}$ are decreased by $\epsilon$ and all $x_{e}, e \in S \cap M^{*}$ are increased by $\epsilon$ then the new solution remains feasible. This new solution will have strictly higher weight than $x$, which is a contradiction. Thus this case cannot occur.

2) $v_{1}$ is a saturated leaf and $v_{2}$ is unsaturated.

An $\epsilon$-perturbation argument like the one above can be used to show that this case too cannot occur.

3) $v_{1}$ is saturated by $M^{*}$. but is not a leaf. $v_{2}$ is either unsaturated, or a saturated leaf.

Since $S$ cannot be extended, it has to be that all edges in $G^{\prime}-M^{*}$ incident to $v_{1}$ have other endpoints in $S$. Let $e$ be one such edge. Then, $e \cap S$ forms a stemmed blossom: the resulting cycle has to be odd, and the remaining part of $S$ will be a stem whose endpoint is $v_{2}$. Note that in this case it has to be that the constraint at $v_{2}$ is loose.

4) Both $v_{1}$ and $v_{2}$ are saturated by $M^{*}$, but are not leaves. Applying the above blossom argument to both $v_{1}$ and $v_{2}$ yields the existence of a blossom pair.

Thus if the LP relaxation is loose then there exists a stemmed blossom or a blossom pair. Now all that is remaining to show is that they are "bad". Let $B_{1}$ be a stemmed blossom in $G^{\prime}$, consisting of blossom $C$ and stem $P$. Then, there exists some $\epsilon>0$ such that if $x_{e}, e \in C \cap M^{*}$ is increased by $\epsilon$, $x_{e}, e \in C-M^{*}$ is decreased by $\epsilon, x_{e}, e \in P \cap M^{*}$ is increased by $2 \epsilon$, and $x_{e}, e \in C-M^{*}$ is decreased by $2 \epsilon$, then the new solution remains feasible for the LP. Also, the new solution weighs

$\epsilon\left[w\left(C \cap M^{*}\right)+2 w\left(P \cap M^{*}\right)-w\left(C-M^{*}\right)-2 w\left(P-M^{*}\right)\right]$

more than $x$. For $x$ to be the unique optimal of the LP, this has to be strictly negative and thus any stemmed blossom $B_{1}$ is bad. A similar argument shows that any blossom pair is bad. This finishes the proof of the proposition.

\section{REFERENCES}

[1] S. M. Aji, G. B. Horn, and R. J. McEliece, "On the convergence of iterative decoding on graphs with a single cycle," in ISIT, 1998, p. 276.

[2] Y. Weiss, "Correctness of local probability propagation in graphical models with loops," Neural Computation, vol. 12, no. 1, pp. 1-41, 2000.

[3] Y. Weiss and W. Freeman, "Correctness of belief propagation in gaussian graphical models of arbitrary topology," Neural Computation, vol. 13, no. 10 , pp. 2173-2200, 2001

[4] D. Malioutov, J. Johnson, and A. Willsky, "Walk-sums and belief propagation in gaussian graphical models," Journal of Machine Learning Research, vol. 7, pp. 2031-2064, Oct. 2006.

[5] T. Richardson and R. Urbanke, "The capacity of low-density parity check codes under message-passing decoding," IEEE Transactions on Information Theory, vol. 47, pp. 599-618, 2001.

[6] P. Rusmevichientong and B. V. Roy, "An analysis of belief propagation on the turbo decoding graph with gaussian densities," IEEE Transactions on Information Theory, vol. 47, no. 2, pp. 745-765, 2001.

[7] Y. Weiss and W. Freeman, "On the optimality of solutions of the max-product belief-propagation algorithm in arbitrary graphs," IEEE Transactions on Information Theory, vol. 47, no. 2, pp. 736-744, Feb. 2001.

[8] M. Bayati, D. Shah, and M. Sharma, "Maximum weight matching via max-product belief propagation," in ISIT, Sept. 2005, pp. 1763 - 1767.

[9] B. Huang and T. Jebara, "Loopy belief propagation for bipartite maximum weight b-matching," in Artificial Intelligence and Statistics (AISTATS), March 2007.

[10] C. Yanover, T. Meltzer, and Y. Weiss, "Linear programming relaxations and belief propagation - an empirical study," Jourmal of Machine Learning Research, vol. 7, pp. 1887-1907, 2006.

[11] M. Wainwright, T. Jaakkola, and A. Willsky, "Map estimation via agreement on (hyper)trees: Message-passing and linear-programming approaches," IEEE Transactions on Information Theory, vol. 51, no. 11, pp. 3697-3717, Nov. 2005.

[12] J. Feldman, D. Karger, and M. Wainwright, "Linear programming-based decoding of turbo-like codes and its relation to iterative approaches." in Allerton Conference on Communication, Control, and Computing, 2002.

[13] J. Feldman, M. Wainwright, and D. Karger, "Using linear programming to decode binary linear codes," IEEE Transactions on Information Theory, vol. 51, pp. 954-972, 2005.

[14] F. Kschischang, B. Frey, and H. Loeliger, "Factor graphs and the sumproduct algorithm," IEEE Transactions on Information Theory, vol. 47, no. 2, pp. 498-519, Feb. 2001.

[15] S. Tatikonda and M. Jordan, "Loopy belief propagation and gibbs measures," in Uncertainty in Artificial Intelligence, vol. 18, 2002, pp. 493-500.

[16] J. Edmonds, "Paths, trees and flowers," Canadian Journal of Mathematics, vol. 17, pp. 449-467, 1965.

[17] D. Bertsekas, "Auction algorithms for network flow problems: A tutorial introduction," Computational Optimization and Applications, vol. 1, pp. 7-66, 1992. 